\title{
Taxonomic identity and occurrence of Garcinia spicata and G. talbotii (Clusiaceae) in peninsular India
}

\author{
Rutuja S. Palkar, M.K. Janarthanam and S. Krishnan* \\ Department of Botany, Goa University, Taleigao Plateau, Goa - 403206, India. \\ *E-mail: skrish@unigoa.ac.in
}

\begin{abstract}
Garcinia talbotii Raizada ex Santapau is often misidentified as G. spicata (Wight \& Arn.) Hook.f. in the regional Floras of northern Western Ghats. This paper clearly delimits these two species with unambiguous morphological and anatomical characters to facilitate authentic identification.
\end{abstract}

Keywords: Distribution, Garcinia spicata, Garcinia talbotii, Misidentification, Northern Western Ghats

\section{Introduction}

Garcinia L. (Clusiaceae) is one of the medicinally and economically important genera due to the presence of two major bioactive compounds namely hydroxycitric acid and garcinol. The genus consists of c. 260 species, and distributed predominantly in tropical regions, especially Asia (Mabberley, 2017). In India, the genus is represented by 39 species and 7 varieties, of which 18 species and 5 varieties are endemic (Maheshwari, 1965; Singh, 1993; Sabu et al., 2013; Nayar et al., 2014; Sarma et al., 2016; Shameer et al., 2017). Garcinia talbotii Raizada ex Santapau is one of the species endemic to Western Ghats that produces edible fruits which can be used in culinary preparations. Quantitative HPLC-PDA analysis of benzophenones and biflavonoids in eight species of Garcinia showed highest amount of xanthochymol in G. spicata (Wight \& Arn.) Hook.f. (Acuna et al., 2012). It also yields timber, used in construction and yellowcoloured pigment from bark and young fruits as a dye.

A critical examination of herbarium specimens of G. spicata housed at BSI, FRLH, CALI, MH, SUK and TBGT revealed that most of them were misidentification of G. talbotii. Similarly, scrutiny of descriptions of G. spicata in the earlier published Floras (Santapau, 1967; Almeida, 1990; Naithani et al., 1997; Godbole \& Das Das, 2000; Yadav \& Sardesai, 2002; Ganeshaiah et al., 2012) that recorded the occurrence of this species in the northern Western Ghats revealed that the diagnostic features provided for G. spicata fall well within the circumscription of G. talbotii. Inclusion of northern Western Ghats, especially Goa and
Maharashtra under the distribution range of G. spicata by other workers (Singh, 1993; Nayar et al., 2014) is also based on the above mentioned earlier works. Further, an examination of fresh field collections corroborates that G. spicata does not occur in northern Western Ghats. The present communication provides detailed description, differentiating characters (Table 1), photographs depicting morphological and anatomical features and images of type specimens to facilitate the accurate identification of both these species.

Garcinia spicata (Wight \& Arn.) Hook.f., J. Linn. Soc., Bot. 14: 486. 1875; Brandis, Indian Trees: 49. 1906; Gamble, Fl. Madras: 53. 1915; Maheshw., Bull. Bot. Surv. India 6: 112. 1965; N.P. Singh in B.D. Sharma \& Sanjappa, Fl. India 3: 125. 1993. Xanthochymus spicatus Wight \& Arn., Prodr. Fl. Ind. Orient. 1: 102. 1834. Type: s.loc., s.die, R. Wight 346A (Syn, E, image!).

Figs. 1,3,5

Tree, dioecious, 5-20 $\mathrm{m}$ high; trunk straight; bark smooth, olive green to brown; latex white; branchlets 4-angled, angles flat. Leaves broadly elliptic-oblong or ovate, 9-22 $\times 4-8 \mathrm{~cm}$, base rounded or obtuse, margins entire, apex retuse, obtuse or acute-acuminate, thick-coriaceous, smooth, dark green; midrib prominent, raised; lateral veins 15-18 pairs, prominent on both surfaces, slightly curved, parallel, clearly visible in dry specimens; petioles to $2 \mathrm{~cm}$ long with stemclasping ligule [anatomy: incurved open vascular strand with large gap]. Flowers in male plant on both spicate and fasciculate inflorescence; spicate inflorescence 2-11-flowered, $1.4-9.5 \mathrm{~cm}$ long; fasciculate inflorescence with $5-15$ short pedicellate flowers arising directly from the stem. Female 


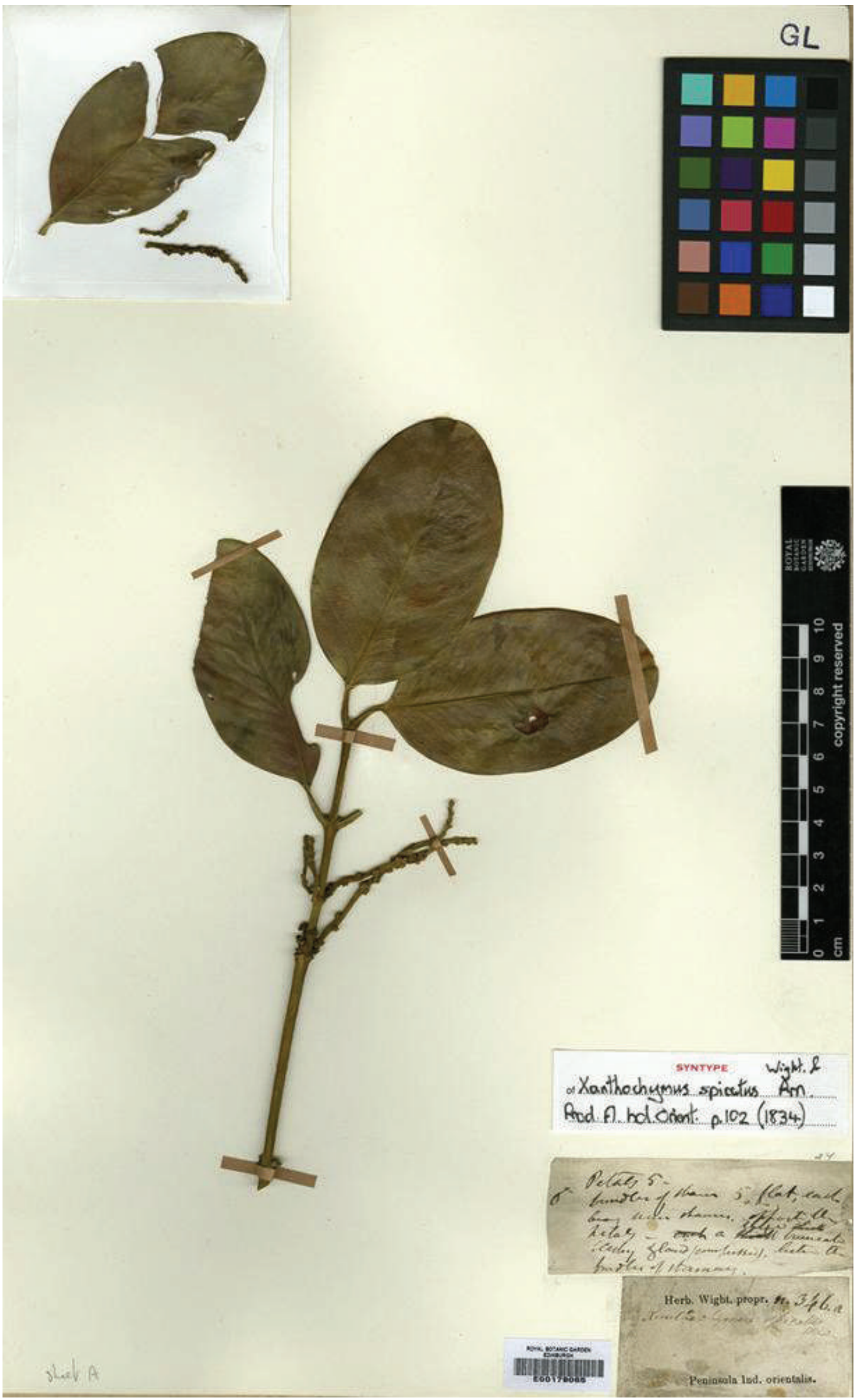

Fig. 1. Syntype of Garcinia spicata (Wight \& Arn.) Hook.f. (三 Xanthochymus spicatus Wight \& Arn. [R. Wight346A, E00179065] @Royal Botanic Garden, Edinburgh. 


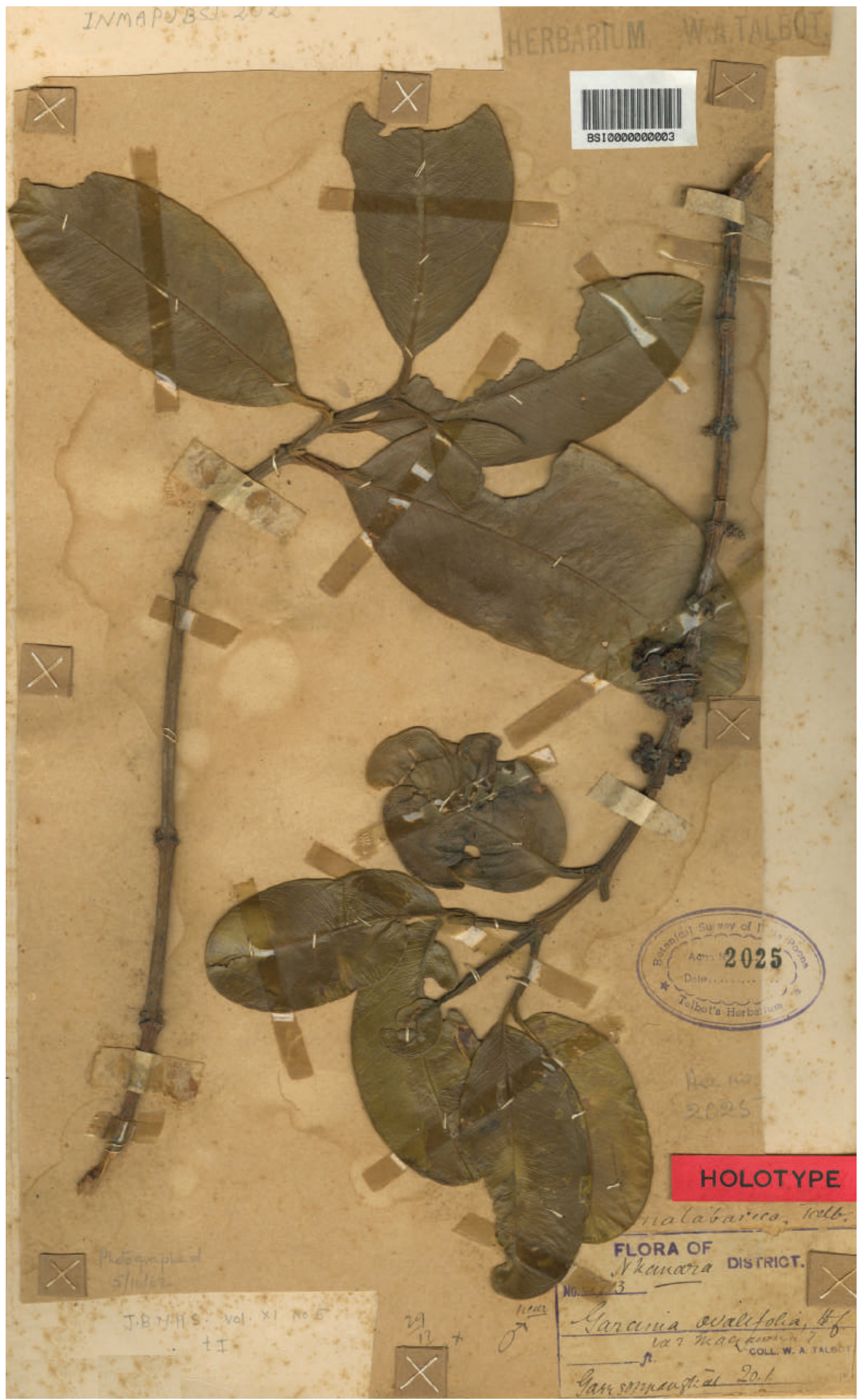

Fig. 2. Holotype of Garcinia talbotii Raizada ex Santapau (G. ovalifolia var. macrantha Vesque) [W.A. Talbot 3713, BSI0000000003] @Botanical Survey of India, Western Regional Centre, Pune. 
flowers solitary or in pairs. Flowers pale green or whitish yellow; sepals 4 or 5 , suborbicular, 2.5-3 $\mathrm{mm}$ long, less than half the length of petal, margins ciliate, membranous, pubescent outside; petals 5, obovate, 6-7 mm long, concave, membranous, pale green or whitish yellow. Male flowers: Pedicels 1-5 $\mathrm{mm}$ long; stamens in 5 spathulate bundles, each bundle with 8-10 stamens, long-clawed, opposite petals; filaments very short, free. Rudimentary pistil absent. Female flowers: Pedicels 1.2-3.5 cm long; staminodes 5, small with weak anthers; ovary oblong, 2-4 locular; style short, c. $1 \mathrm{~mm}$ long; stigma 3-5-lobed, peltate, spreading, $2-3 \mathrm{~mm}$ in diameter, persistent, thick at peltate region, black. Fruits broadly globose, subglobose or depressedglobose, shallowly angular, to $4 \mathrm{~cm}$ in diameter; immature fruits with mild ridges and furrows, pale green; ripened fruits smooth, yellow, with mild unpleasant odour; pulp acidic, bitter taste; seeds $1-3$, broadly oblongoid, 1.5-2.5 × 1-1.3 cm, dark brown, black when dried.

Flowering \& fruiting: January-August.

Distribution: India (Andhra Pradesh, Karnataka, Kerala and Tamil Nadu) and Sri Lanka.

Specimens examined: INDIA, Andhra Pradesh: Nellore district, Sriharikota forest, May 1904, s.coll. 3034, 3035 (MH). Karnataka: Agumbe district, Barakana, Balehalli, 19.5.1960, R. Sundara Raghavan 62720 (BSI). Kerala: Kasargod district, Cherpady, Uduma, 12.3.1993, A. Nazarudeen 16408 (TBGT); Kollam district, Karingalodathodu area, Tenmalai range, 7.12.1961, K.N. Subramanian 77529 (BSI); Kottayam district, Koruthode, 200 m, 12.11.1995, Jomy Augustine 16906 (CALI); Puthupalli, s.die, G.S. Puri 36572 (BSI); Palakkad district, Mukkali forest, 7.3.1975, E. Vajravelu 46240 (MH); Pathanamthitta district, Pamba, 200 m, 12.2.1994, Jomy Augustine 13370 (CALI); Pamba, 18.3.1994, A. Nazarudeen 19587 (TBGT). Tamil Nadu: Cuddalore district, Puthupet, 1203' N, 7952' E, 5 m, 18.5.2017, R.S. Palkar \& S. Krishnan 5177 (Goa University Herbarium); Pudukkottai district, Pudukkottai, November 1909, C.A. Barber 8498 (MH); Villupuram district, Marakkanam, 3.6.1907, C.A. Barber 8321 $(\mathrm{MH}) ;$ Marakkanam, Kurumbaram Reserve Forest, $12^{\circ} 12^{\prime} \mathrm{N}, 7^{\circ} 56^{\prime} \mathrm{E}, 10 \mathrm{~m}, 5.5 .1995$, J.D.H., P.B. \& W.F.G. 02757 (FRLH). SRI LANKA, Ceylon Southern Province: Hambantota district, Yala, Talgasmankade, 26.4.1973, L.H. Cramer 4124 (MH).

Garcinia talbotii Raizada ex Santapau, Rec. Bot. Surv. India 16(1): 14. 1960; Maheshw., Bull. Bot. Surv. India 6: 113. 1965; N.P. Singh in B.D. Sharma \& Sanjappa, Fl. India 3: 127. 1993; M.R. Almeida, Fl. Maharashtra 1: 88. 1996; Godbole \& Das Das in
N.P. Singh \& Karthik., Fl. Maharashtra 1: 277. 2000. G. ovalifolius (Roxb.) Hook.f. ex T. Anderson var. macrantha T. Anderson in Hook.f., Fl. Brit. India 1: 269. 1874. G. spicata (Wight \& Arn.) Hook.f. var. macrantha Vesque in A. DC., Monogr. Phan. 8: 311. 1893; T. Cooke, Fl. Bombay 1: 79. 1901. G. malabarica Talbot, J. Bombay Nat. Hist. Soc. 11: 234, 1897, non Desr. 1792. Xanthochymus ovalifolius J. Graham, Cat. Pl. Bombay: 26. 1839, non Roxb. 1832.

Garcinia spicata auct. non (Wight \& Arn.) Hook.f. 1875: T. Cooke, Fl. Bombay 1: 78. 1901; Santapau, Rec. Bot. Surv. India 16(1): 14. 1967, incl. spec. Blatter 624; S.M. Almeida, Fl. Savantwadi 1: 54. 1990, incl. spec. G.A. Gammie 15025; H.B. Naithan et al., Forest Fl. Goa: 65. 1997; Godbole \& Das Das in N.P. Singh \& Karthik., Fl. Maharashtra 1: 276. 2000.

Type: INDIA, Karnataka: North Kanara district, Garrsoppah Ghat, 20.1.18??, W.A. Talbot 3713 (holo, BSI0000000003, image!).

Figs. 2,4,5

Tree, dioecious, 6-18 m high; trunk straight; bark smooth, olive green; latex sticky, turning into brownish; branches angular, slender, twiggy; branchlets 6-angled, angles concave. Leaves elliptic-oblong or ovate, $5-18 \times 4-9 \mathrm{~cm}$, base rounded, margins entire, apex obtuse, retuse or acute-acuminate, coriaceous, shiny, dark green; midrib prominent; lateral veins $16-18$ or more pairs, distinct, parallel; petioles to $1.8 \mathrm{~cm}$ long [anatomy: curved open vascular strand with no gap or minute gap]. Spikes in male plant on old wood, numerous, 5-8 mm long; spikes absent in female plant. Flowers 3-12, on fasciculate inflorescence in both male and female plant. Flowers creamywhite, white to greenish-yellow; sepals 5, orbicular, concave, green, $3-5 \mathrm{~mm}$ across; petals 5 , orbicular or suborbicular, concave, $c .10 \times 8 \mathrm{~mm}$, pale green or whitish yellow. Male flowers: Pedicels 5-8 $\mathrm{mm}$ long; stamens in 5 spathulate bundles, thick, 8-12 anthers on each phalange, reddish, opposite petals; filaments very short, free. Rudimentary pistil absent. Female flowers: Pedicels $0.4-1 \mathrm{~cm}$ long; staminodes 5 , thin, flattened bundles, 4 or 5 anthers on each phalange, similar to male flower; ovary globose, 2 or 3-locular; stigma 3-lobed, 1-3 $\mathrm{mm}$ in diameter, narrow at base, persistent, thick, black. Fruits globular, 4-6 cm; immature fruits smooth, without any ridges and furrows, dark green; ripened fruits smooth, yellow, with bad odour; pulp acidic, bitter; seeds 1 or 2 , narrowly oblongoid, $2.5-3 \times 1.2-1.5 \mathrm{~cm}$, brown.

Flowering \& fruiting: January-June.

Distribution: India (Goa, Karnataka, Kerala and Maharashtra). Endemic. 

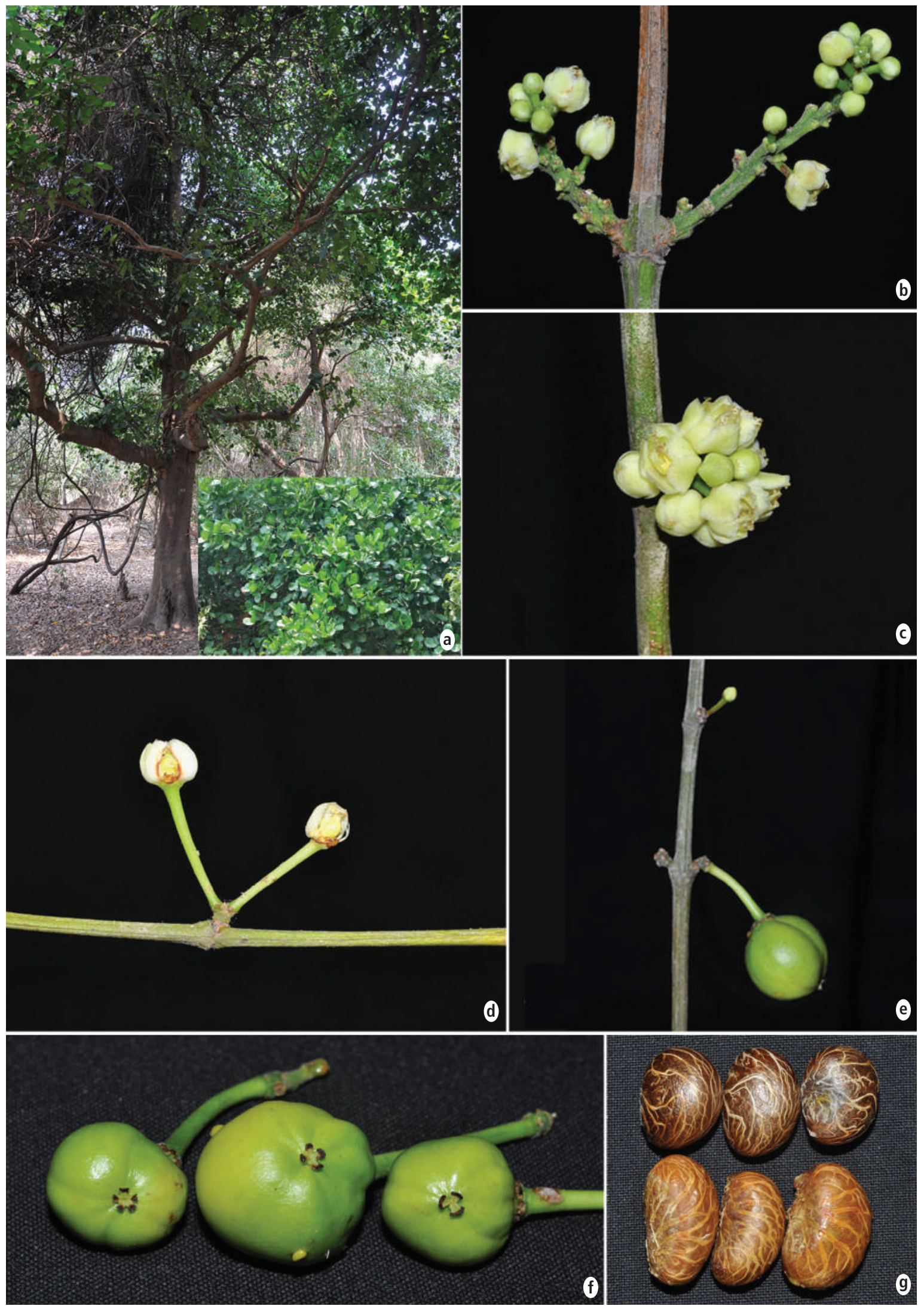

Fig. 3. Garcinia spicata Hook.f.: a. Habit (Insert: close-up of branch); b. Spicate inflorescence (male); c. Fasciculate inflorescence (male); d. Female flower; e. Immature fruit with ridges and furrows; f. Fruits with 3-5 stigmatic lobes; g. Matured and dried seeds: upper row - G. spicata, lower row - G. talbotii. 


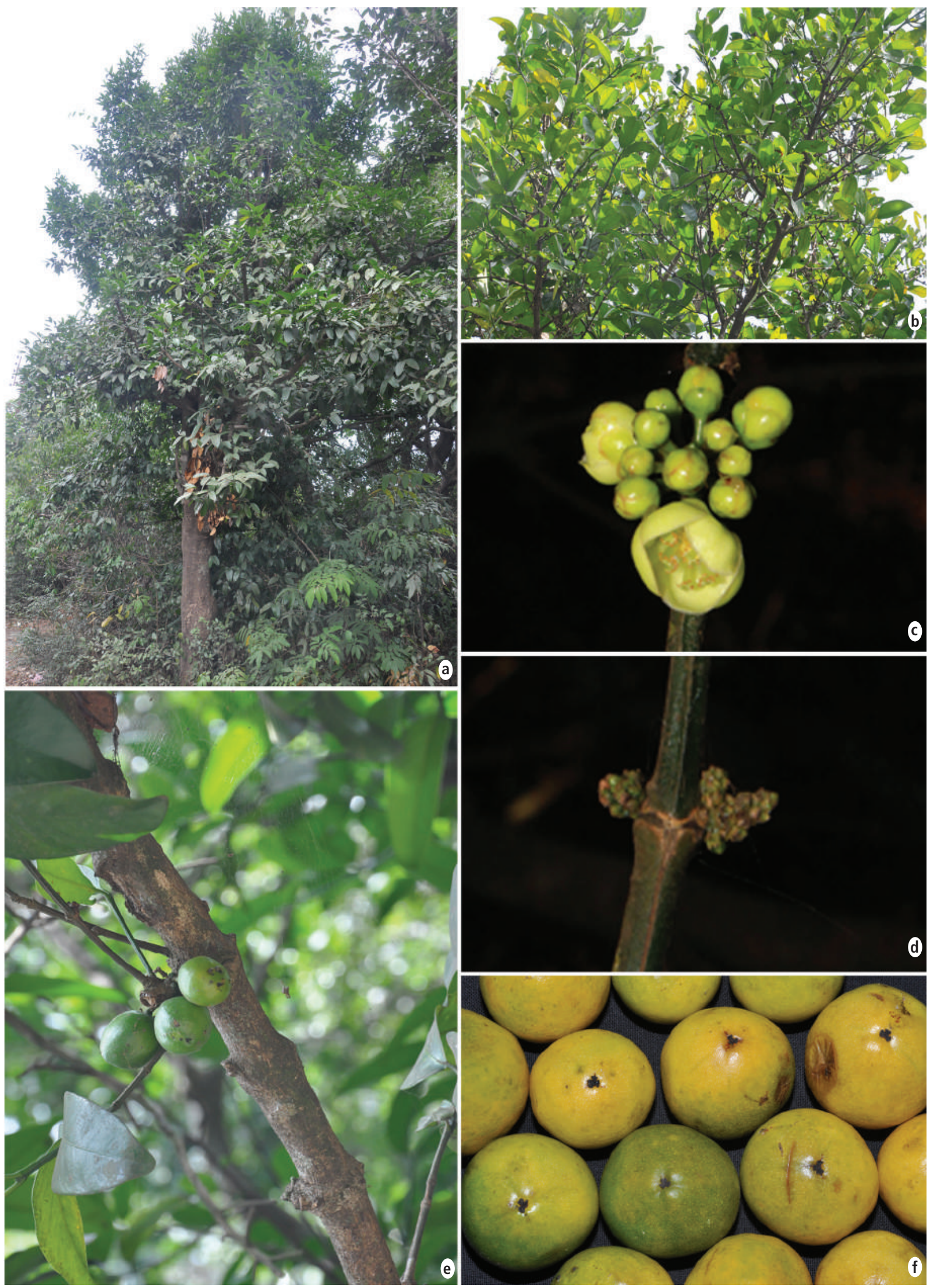

Fig. 4. Garcinia talbotii Raizada ex Santapau: a. Habit; b. Branch showing numerous spikes in male plant; c. Fasciculate inflorescence (male); d. Close-up of spike; e. Immature fruit with smooth surface; f. Mature fruits showing 3-lobed stigma. 


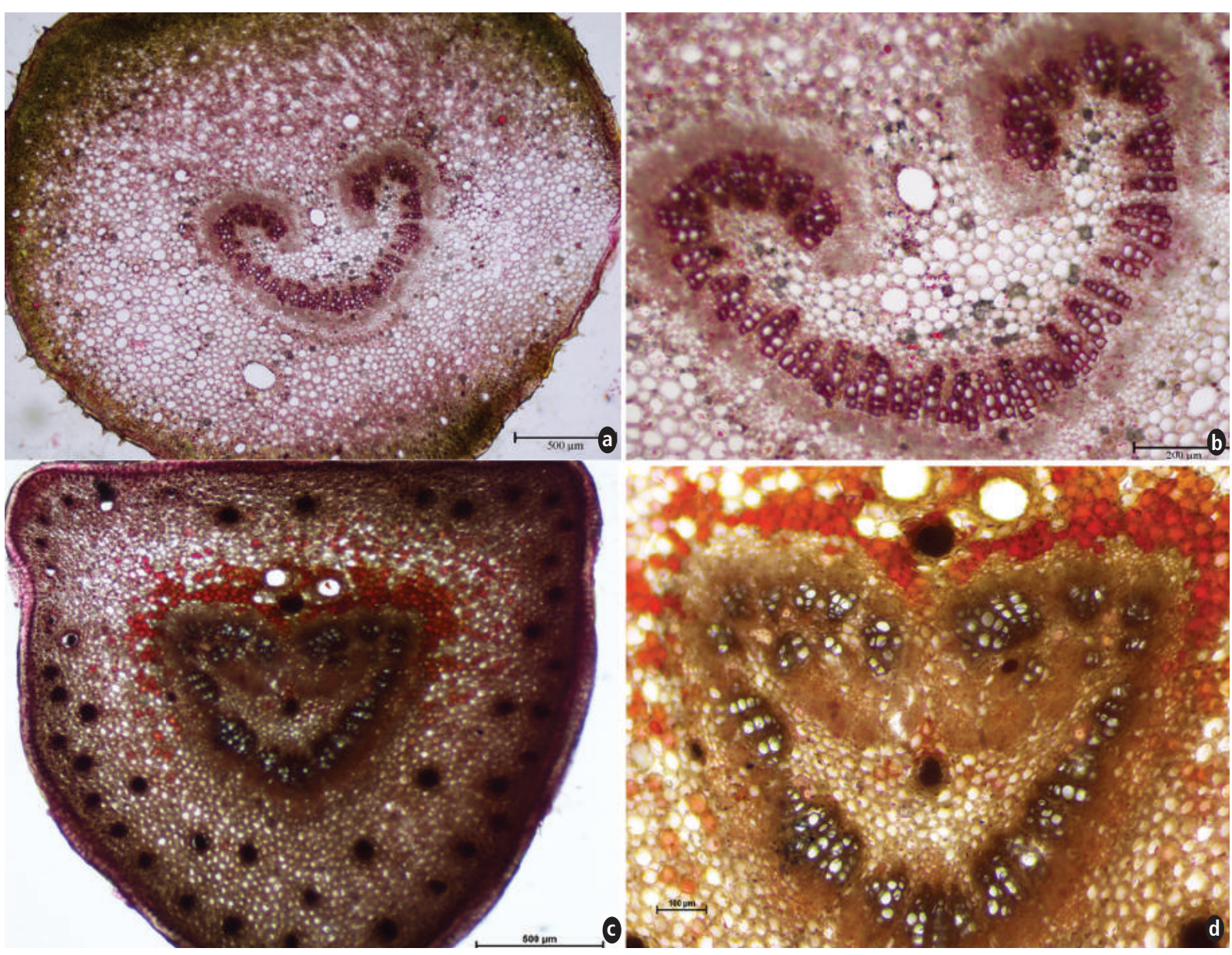

Fig. 5. T.S. of petioles stained with safranin: a,b. Garcinia spicata showing incurved open vascular strand with large distinct gap; c,d. Garcinia talbotii with curved vascular strand with very minute gap.

Table 1. Comparative key characteristics of Garcinia spicata and G. talbotii

\begin{tabular}{|c|c|c|}
\hline Characters & G. spicata & G. talbotii \\
\hline Branchlets & 4-angled; angles flat & 6-angled; angles concave \\
\hline Flowers in male plant & $\begin{array}{l}\text { Spicate and fasciculate. Spikes } \\
1.4-9.5 \mathrm{~cm} \text { long }\end{array}$ & $\begin{array}{l}\text { Fasciculate. Also arranged in } \\
\text { numerous pseudospikes of } \\
0.5-0.8 \mathrm{~cm} \text { long }\end{array}$ \\
\hline Flowers in female plant & Solitary or in pairs & Fasciculate \\
\hline $\begin{array}{l}\text { Length of pedicels in female } \\
\text { flowers }\end{array}$ & $1.2-3.5 \mathrm{~cm}$ & $0.4-1 \mathrm{~cm}$ \\
\hline Ovary & 2-4-locular & 2 or 3 -locular \\
\hline Stigmatic lobes & $3-5$ & 3 \\
\hline Fruits & Shallowly angular & Smooth \\
\hline Seeds & $\begin{array}{l}\text { Broadly oblongoid, } 1.5-2.5 \times 1-1.3 \\
\mathrm{~cm} \text {, dark brown, black when dry }\end{array}$ & $\begin{array}{l}\text { Narrowly oblongoid, } \\
2.5-3 \times 1.2-1.5 \mathrm{~cm} \text {, brown }\end{array}$ \\
\hline Vascular strand in petiole & Incurved open with large gap & $\begin{array}{l}\text { Curved open with no gap or } \\
\text { minute gap }\end{array}$ \\
\hline
\end{tabular}


Specimens examined: INDIA, Goa: North Goa district, Vagheri hills, 19.12.1997, Vaishali C. Joshi 1296 (Goa University Herbarium). Karnataka: Belgaum district, Hemadga, s.die, Nilesh $V$. Malpure NVM 3766 (SUK); Sada plateau, $15^{\circ} 40.18^{\prime}$ N, 74⒎10’ E, $806 \mathrm{~m}, 8.5 .2016$, R.S. Palkar 5172 (Goa University Herbarium). Kerala: Palakkad district, Nelliampathy ghat, 29.6.1914, S.T. Dunn s.n. (MH Acc. No. 3018). Maharashtra: Kolhapur district, Amba, s.die, Priti Mahekar SUK-392 (SUK); Gajapur, s.die, Milind Sardesai 831 (SUK); Manoli, Amba, 11.5.1904, R.K. Bhide s.n. (BSI); Palghar district, Shirgaon, 21.4.1957, L.A. Vasavada 17031 (BSI); Pune district, Auhpe forest, 23.3.1907, G.M. Ryan 1751* (BSI); Bhimashankar, 25.10.1956, L.A. Vasavade 8575* (BSI); Bhimashankar, 13.6.1961, K.P. Janardhanan 72256 (BSI); Bhimashankar, 7.6.1962, K.P. Janardhanan 76642 (BSI); Bhimashankar, s.die, J.A.V. 9314* (BSI); Choura hill, Bhimashankar, 24.2.1961, K.P. Janardhanan 69201 (BSI); Choura hill, Bhimashankar, 22.4.1961, K.P. Janardhanan 72108 (BSI); Choura hill, Bhimashankar, 4.4.1962, K.P. Janardhanan 76581 (BSI); Bhimashankar, 2.2.1999, S.R. Yadav 5845 (SUK); Raigad district, Matheran, 2.11.1907, H.P. Paranjpe s.n.* (BSI Acc. No. 6751); Matheran, 9.11.2006, S.C. Majumdar E S.K. Das Das 192443 (BSI); Sudagarh Fort, Pali, 9.3.1979, M.J. Kothari 156423 (BSI); Satara district, Khandala, 21.3.1903, G.A. Gammie 16163* (BSI); Koyna, 23.11.1978, R.K. Kochhar 157811 (BSI); Jaigad, Koyna, 11.2.1979, R.K. Kochhar 158332* (BSI); Kusavade, May 1995, M.P. Bachulkar Cholekar 20040* (SUK); Vasota, April 1993, M.P. Bachulkar Cholekar 5550 (SUK); Sindhudurg district, Amboli, 28.4.1902, G.A. Gammie 15025* (BSI); Amboli, $15^{\circ} 56.28^{\prime} \mathrm{N}, 73^{\circ} 59.99^{\prime} \mathrm{E}, 821 \mathrm{~m}, 27.5 .2015$, R.S. Palkar 5173 (Goa University Herbarium); Chaukul, Amboli, 9.11.1965, B.G. Kulkarni 107936 (BSI). (*Specimens of G. talbotii wrongly identified as G. spicata).

\section{Acknowledgements}

Authors gratefully acknowledge the financial support provided by the CSIR (No. 38(1348)/13/ EMR-II), New Delhi, to carry out the research work. They are thankful to the Scientist/Professorin-Charge of BSI, CALI, FRLH, MH, SUK and TBGT, for providing permission to consult the herbarium specimens. Authors are thankful to the authorities of BSI and E for permission to reproduce type images. Authors are grateful to: Dr. N. Parthasarathy, Professor, Department of Ecology and Environmental Sciences, for his help in the collection of G. spicata, Dr. Sharad S. Kambale, Assistant Professor, Department of Botany, MVP
Samaj's Arts, Commerce and Science College, Nashik, Maharashtra, for his help and suggestions and Ms. Diksha D. Naik, Department of Botany, Goa University, for her help in anatomical studies.

\section{Literature Cited}

Acuna, U.M., Keyvan, D., Basile, M.J. \& Kennelly E.J. 2012. Quantitative high-performance liquid chromatography photo-diode array (HPLC-PDA) analysis of benzophenones and biflavonoids in eight Garcinia species. J. Food Compos. Anal. 25(2012): 215-220. http:// dx.doi.org/10.1016/j.jfca.2011.10.006.

Almeida, S.M. 1990. The Flora of Savantwadi. Vol. 1. Scientific Publishers, Jodhpur. pp. 53-55.

Cooke, T. 1901. The Flora of the Presidency of Bombay Vol. 1. Taylor \& Francis, London. pp. 75-82.

Ganeshaiah, K.N., Ganesan, R., Vasudeva, R., Kushalappa, C.G., Menon, A.R.R., Patwardhan, A., Yadav, S.R. \& R.U. Shaanker 2012. Plants of the Western Ghats. Vol. 1. School of Ecology and Conservation, University of Agriculture Sciences, Gandhi Krishi Vignana Kendra, Bengaluru. pp. 339-344.

Godbole, A. \& S.K. Das Das 2000. Clusiaceae. In: Singh, N.P. \& S. Karthikeyan (eds.), Flora of Maharashtra State. Dicotyledones. Vol. 1. Botanical Survey of India, Calcutta. pp. 273283.

Mabberley, D.J. 2017. Mabberley's Plant-Book: A portable dictionary of plants, their classification and uses. Fourth Edition. Cambridge University Press, Cambridge. p. 378.

Maheshwari, J.K. 1965. Taxonomic studies on Indian Guttiferae III. The Genus Garcinia Linn. s.l. Bull. Bot. Surv. India 6(2-4): 107-135.

Naithani, H.B., Sahni, K.C. \& S.S.R. Bennet 1997. Forest Flora of Goa. International Book Publishers, Dehra Dun. pp. 61-66.

Nayar, T.S., Sibi, M. \& A. Rasiya Beegam 2014. Flowering Plants of the Western Ghats. Vol. 1. Dicots. Jawaharlal Nehru Tropical Botanic Garden and Research Institute, Thiruvananthapuram. pp. 274-277.

Sabu, T., Mohanan, N.N., Krishnaraj, M.V.N., Shareef, S.M., Shameer, P.S. \& P.E. Roy 2013. Garcinia pushpangadaniana (Clusiaceae), a new species from the southern Western Ghats, India. Phytotaxa 116(2): 54-56. http://dx.doi. org/10.11646/phytotaxa.116.2.2. 
Santapau, H. 1967. The Flora of Khandala on the Western Ghats of India. Third Revised Edition. Rec. Bot. Surv. India 16(1): 1-372.

Sarma, J., Shameer, P.S. \& N.N. Mohanan 2016. A new species of Garcinia (Clusiaceae) from Assam, North East India. Phytotaxa 252(1): 73-76. http://dx.doi.org/10.11646/ phytotaxa.252.1.8.

Shameer, P.S., Sabu, T. \& N.N. Mohanan 2017. Garcinia gamblei (Clusiaceae), a new species from the southern Western Ghats, India.
Phytotaxa 297(1): 71-76. http://dx.doi. org/10.11646/phytotaxa.297.1.7.

Singh, N.P. 1993. Clusiaceae. In: Sharma, B.D. \& M. Sanjappa (eds.), Flora of India. Vol. 3. Botanical Survey of India, Calcutta. pp. 86-151.

Yadav, S.R. \& M.M. Sardesai 2002. Flora of Kolhapur District. Shivaji University, Kolhapur. p. 63.

Received: 13.8.2017

Revised and Accepted: 29.12.2017 